\title{
Un savoir-faire implicite des enseignants : adapter leurs questions au niveau de leurs élèves
}

\author{
Audrey Murillo \\ Maître de conférences à l'École Nationale de Formation Agronomique (ENFA), Toulouse
}

\begin{abstract}
Résumé
Nous nous intéressons aux produits d'apprentissages informels : les savoir-faire implicites qui ont été construits par des enseignants expérimentés. Nous présentons notre cadre théorique, issu du constructivisme et de l'ergonomie de langue française, et l'opérationnalisons par le biais du concept de schème (Vergnaud, 1985). Dans ce but, nous adossons notre méthodologie à des observations répétées d'enseignants (et non des verbalisations). Nous cherchons à mettre au jour des savoir-faire relatifs au pilotage de la classe : quels choix les enseignants font-ils lorsqu'ils prescrivent une tâche à leurs élèves et leur posent des questions pour avancer dans la résolution de cette tâche ? Nous montrons que les enseignants parviennent à poser des questions qui conduisent à un taux de réussite acceptable à la fois de la part de la classe et de chacun des élèves, ce qui n'est pas sans lien avec leur mobilisation au travail.
\end{abstract}

\section{Implicit constructs in the teaching profession's learning}

This chapter deals with the products of informal learning: the implicit knowledge that have been developed by experienced teachers. We present our theoretical framework, derived from constructivism and ergonomics, and operationalize it through the concept of scheme (Vergnaud, 1985). For this purpose, we make repeated observations of teachers. We bring to light knowledge relating to the piloting of the class: what choices do teachers when they prescribe a task to their students and ask them questions to solve this task? We show that teachers are able to ask questions that lead to a success rate acceptable both on the part of the class and individual students.

\section{Introduction}

L'activité d'enseignement se caractérise par la complexité de l'environnement dans lequel elle se déroule. Doyle (1986) en énumère quelques caractéristiques : la multidimensionnalité, la simultanéité, l'immédiateté, l'imprévisibilité, le caractère public et l'historicité. Avec une approche ergonomique, à l'instar de Rogalski (2003), nous considérons que l'enseignant doit gérer dans sa classe un environnement dynamique humain : cet environnement évolue même en absence d'action de l'enseignant, et de façon d'autant plus complexe qu'il est composé, entre autres, d'êtres humains (qui sont ici les élèves). La gestion de cet environnement complexe peut être en partie enseignée, en formation initiale ou continue. Nous postulons également, en suivant Maurice (2006), qu'une part des savoir-faire des enseignants est implicite, et relève d'apprentissages informels. Ces derniers peuvent être appréhendés par leurs processus (la manière d'apprendre, les périodes durant lesquelles on apprend...) ou par leurs produits, qui résultent des processus (Carré, 2005). Notre contribution a pour objectif de mettre au jour des produits d'apprentissages informels : des savoir-faire implicites mis en œuvre par les enseignants, relatifs au pilotage de leur classe, et pas nécessairement enseignés en formation des maîtres. Notre analyse porte sur les pratiques de cinq enseignants lors de séances de découverte de texte, au Cours Préparatoire. Quels choix les enseignants expérimentés font-ils lorsqu'ils prescrivent une tâche à leurs élèves et qu'ils leur posent des questions pour avancer dans la résolution de cette tâche ? En quoi ces choix peuvent-ils faciliter la participation et l'implication de chaque élève dans la tâche ?

Dans un premier temps, nous présenterons notre approche théorique, issue des principes du constructivisme et de l'ergonomie de langue française. Après avoir exposé nos choix méthodologiques pour mettre au jour des savoir-faire implicites d'enseignants expérimentés, nous présenterons un savoir-faire en relation à la fois avec les dimensions de "gestion de la classe » et de «gestion du savoir » : les enseignants expérimentés parviennent à choisir des tâches pour leurs élèves et à leur poser des questions qui les conduisent à un taux de réussite 
acceptable, à la fois de la part de la classe dans sa globalité, et de chacun des élèves en particulier.

\section{De nombreux savoir-faire des enseignants sont implicites}

Pour définir les «savoir-faire implicites » des enseignants, nous pouvons nous appuyer momentanément sur ce que Remoussenard (2014) nomme les «dimensions cachées » de l'activité : des dimensions qui échappent aux référentiels, à la formation, aux institutions... Étudier ces dimensions nous paraît tout à fait pertinent pour approcher les «savoirs de l'activité » des professionnels. Ces savoirs sont «liés au contexte et aux conditions spécifiques de mise en œuvre de l'activité » et diffèrent des «savoirs de la tâche », qui sont souvent répertoriés, organisés et énoncés (Savoyant, 2008). Cela rencontre pleinement nos préoccupations ; toutefois, notre approche se distingue de celle de Remoussenard en deux sens :

- tout d'abord, dans le sens où le qualificatif «caché » implique une action intentionnelle : une personne cache des dimensions de son travail à d'autres personnes (sa hiérarchie, ses pairs, un chercheur...). Nous nous intéressons ici plutôt aux dimensions qui peuvent être insues de la personne même qui les met en œuvre.

- Enfin, dans le sens où ce ne sont pas des comportements implicites qui sont l'objet de notre étude, mais plutôt l'organisation (invisible) de comportements visibles.

Il s'agit donc pour nous d'approcher l'organisation de savoir-faire construits implicitement par les enseignants.

Hors du domaine du travail enseignant, prenons un exemple d'organisation de savoir-faire construit implicitement, qui n'est pas nécessairement conscientisé par les sujets qui l'ont développé, mais qui a été mis au jour par un regard distancié. Dans le domaine moteur, un savoir-faire paraissant aussi trivial que celui de la marche peut être déconstruit de manière très fine. Les biomécaniciens ont par exemple déterminé que la phase où le pied est en contact avec le sol dure $60 \%$ de la durée d'un cycle de marche et est décomposée en cinq sousphases : frappe du talon, plantigrade, mi-appui, décollement du talon, digitigrade (Ducroquet, 1965). Le marcheur expérimenté saura peut-être, au mieux, évoquer le rythme et la longueur de ses pas en fonction de la vitesse qu'il souhaite atteindre, les obstacles qu'il doit éviter... mais son analyse ne sera pas celle du biomécanicien :

- d'une part parce qu'il ne possède pas son vocabulaire (le marcheur n'a pas besoin de savoir que le signifiant « digitigrade » correspond à la poussée des orteils) ;

- d'autre part, et surtout, le signifié de « digitigrade » n'est pas plus utile au marcheur que le signifiant : il est tout à fait possible de (bien) marcher durant toute une vie sans prendre conscience que ses orteils effectuent une poussée en milieu du cycle de marche. Ce n'est donc pas, bien sûr, qu'une question de vocabulaire.

Les savoirs et savoir-faire utiles au marcheur ne sont pas ceux issus des études en biomécanique, et réciproquement, le biomécanicien qui a une très bonne connaissance de la marche peut lui-même être boiteux...

L'organisation des savoir-faire liés à la marche sont donc difficilement explicitables sans un regard extérieur outillé. Qu'en est-il alors des savoirs et savoir-faire liés à l'activité d'enseignement ? Ils sont sans nul doute bien plus variés et complexes que ceux qui sont liés à la marche. Une partie est explicite, enseignable, transmissible. Nous émettons toutefois l'hypothèse que l'organisation sous-jacente de nombreux savoir-faire est encore non explicitée. Nous allons tenter dans cette contribution de mettre en lumière une organisation (invisible) de comportements (visibles) d'enseignants expérimentés. 


\section{Quels choix théoriques pour approcher des savoir-faire implicites ?}

Afin d'approcher des savoir-faire implicites d'enseignants expérimentés, nos choix théoriques s'articulent autour de trois propositions, issues des théories constructivistes.

\section{- L'activité des professionnels ne se limite pas aux prescriptions qu'ils reçoivent.}

C'est un des postulats fondateurs de l'ergonomie de tradition française. En psychologie ergonomique, la tâche est ce qu'il y a à faire ; l'idée de prescription, voire d'obligation, est sous-jacente à ce concept (Leplat \& Hoc, 1983). La tâche prescrite est « celle qui figure dans les instructions, les consignes, les procédures » (Leplat, 2000). L'activité est ce qui est mis en œuvre pour réaliser la tâche (Leplat \& Hoc, 1983). Rabardel, Carlin, Chesnais, Lang et Pascal (1998) attribuent plusieurs origines à l'écart entre prescriptions et activité réalisée :

- il est impossible de prescrire le travail dans tous ses détails : le travail réel excède donc le prescrit ;

- les professionnels peuvent avoir des difficultés à suivre une prescription trop floue ;

- les professionnels doivent prendre en compte la singularité des situations qu'ils rencontrent, et s'éloigner ainsi des prescriptions ;

- les professionnels prennent en compte leurs propres objectifs et systèmes de valeurs.

L'écart entre travail prescrit et travail effectif peut donc être créé intentionnellement ou non par le professionnel. Notons que cet écart ne constitue pas obligatoirement une « erreur »dans le travail : il est souvent nécessaire, et dû à une adaptation aux contraintes des situations rencontrées. Six (1999) évoque les «prescriptions remontantes » que le professionnel doit également prendre en compte: un élève qui pose une question, un groupe d'élèves qui bavarde... appellent certains types d'actions de l'enseignant, qui répondent ou non à ces prescriptions particulières, venues de la situation professionnelle elle-même et non d'instructions en amont. Cela explique qu'un enseignant ne peut suivre strictement une méthode pensée a priori, mais doit nécessairement l'adapter en cours d'enseignement (Bru, 1991).

\section{- La réussite précède la compréhension.}

Dans son ouvrage «Réussir et comprendre », Piaget (1974) montre que l'atteinte d'un but (la réussite en action) précède souvent la conscientisation des raisons de la réussite (la compréhension). La connaissance nécessaire à la réussite n'est pas la même que celle qui est nécessaire à la compréhension. Pour Vergnaud (1999, p. 8), «les décalages entre action et explicitation, entre réussite et compréhension, entre cognitif et métacognitif existent dans tous les domaines d'activité. Ils concernent les adultes comme les enfants, les activités intellectuelles supérieures comme les activités réputées plus simples, les gestes notamment ».

Qu'il s'agisse d'enseigner ou de réaliser une autre activité, l'expérience se construit ainsi en action, et passe par des savoir-faire peu réflexifs, avant qu'une abstraction réfléchissante puisse avoir lieu (Maurice, 2006).

- La construction de schèmes permet l'adaptation aux situations professionnelles. À la suite de Piaget, la théorie des champs conceptuels développée par Vergnaud (1985) explique les processus sous-jacents à l'activité au moyen du concept de schème. Le schème est « une organisation invariante de l'activité pour une classe de situations » (Vergnaud, 2001, p.40). L'auteur précise que « ce qui est invariant, c'est l'organisation, non pas l'activité et la conduite » (Vergnaud \& Récopé, 2000, p.45). La plupart des schèmes ne sont donc pas 
associés à des automatismes ni des routines ; ils permettent «l'adaptation de l'activité et de la conduite aux valeurs différentes prises par les variables de situation » (op. cit.).

Cette théorie permet de ne pas dissocier, théoriquement et méthodologiquement, l'activité observable et la cognition. Elle se démarque donc, comme l'écrit Maurice (2007) :

- du paradigme «processus-produit », qui est centré sur des observables et occulte la cognition des enseignants et des élèves ;

- du paradigme de la «pensée des enseignants », qui tente d'accéder à leur cognition par des verbalisations et ce faisant, met au second plan leur comportement.

L'étude des conceptualisations en acte permet de trouver une alternative à ces deux courants ; en posant que l'action et les conceptualisations sont interdépendantes, Vergnaud (1996) reconnaît la richesse de l'action tout en étant « suffisamment béhavioriste " ${ }^{1}:$ il dépasse à la fois « l'obstacle mentaliste, des concepts sans corps », et "l'obstacle physiciste, des corps sans concepts » (Sensevy, 2007, p. 43).

D'après la théorie des champs conceptuels, un enseignant expérimenté serait un enseignant qui s'est adapté aux situations de classe en construisant des schèmes. Ces schèmes lui permettraient d'être efficace dans des situations habituelles (par assimilation), tout comme dans des situations nouvelles (par accommodation); ainsi, le concept de schème permet de rendre compte des invariances des pratiques tout comme de leurs adaptations nouvelles (Pastré, 2011).

\section{Problématique : quels schèmes les enseignants construisent-ils vis-à-vis du niveau de difficulté des questions posées à leurs élèves ?"}

Nous cherchons à appréhender les schèmes des enseignants concernant le niveau de difficulté des tâches qu'ils prescrivent à leurs élèves. Nous pensons en effet que les choix des enseignants quant à cette dimension participent au pilotage de la classe. À propos des pratiques des enseignants, de précédents travaux portant sur les tâches évaluatives (Posthumus, 1947 ; Grisay, 1984 ; Crahay, 1996 ; Bressoux \& Pansu, 2003) montrent que les choix des enseignants quant au niveau de difficulté de ces tâches ont souvent des caractéristiques communes dont les enseignants ne sont pas nécessairement conscients :

- d'une part, les évaluations créées par les enseignants discriminent davantage leurs élèves que les évaluations utilisées par les enquêteurs, car les premières n'évaluent ni ce qui est connu de toute la classe, ni ce que toute la classe ignore ;

- d'autre part, les enseignants ajustent le niveau de difficulté des tâches évaluatives pour parvenir à une distribution gaussienne des notes, similaire d'une année à l'autre.

Les enseignants auraient donc construit des savoir-faire leur permettant d'ajuster le niveau de difficulté des tâches évaluatives au niveau de leur classe. Nous nous interrogeons dans ce texte sur ce qu'il en est des tâches d'apprentissage, sans évaluation formelle : les contraintes dans la prescription de ces tâches sont-elles moindres, car la dimension «évaluation » y est quasi-inexistante, ou des contraintes communes à l'ensemble des tâches prescrites (avec évaluation formelle ou non) existent-elles ? Nous avons montré dans de précédents travaux, au moyen de l'indicateur de «Distance à la Performance Attendue » (Maurice \& Murillo, 2008 ; Murillo, 2010), que les tâches d'apprentissage choisies par les enseignants à chaque séance plaçaient toujours...

\footnotetext{
${ }^{1}$ Par " suffisamment béhavioriste ", Sensevy, en suivant Tiercelin (2002), indique que Vergnaud se détache du psychologisme en étudiant les significations à partir " des comportements ou des dispositions à l'action » et non de « ce qui est dans la tête ».
} 
- ...certains élèves en difficultés, dans le sens où ces derniers, deux jours après la séance, n'étaient pas capables d'utiliser les savoirs et savoir-faire faisant l'objet de la séance observée ;

- ...certains élèves en situation de réussite, dans le sens où deux jours après la séance, ils étaient capables d'utiliser la plupart des savoirs et savoir-faire faisant l'objet de la séance observée (soit parce qu'ils les avaient déjà construits, soit parce que la séance leur avait permis de les construire).

Ces études portaient sur des séances de découverte de texte, au Cours Préparatoire. Nous avons ensuite souhaité nous centrer sur les performances des élèves durant ces tâches de découverte de texte. En effet, même si tous les élèves d'une classe n'ont pas des opportunités d'activité identiques lors de la séance, tous ou presque doivent pouvoir, en cours de séance, entrer dans le jeu de découverte de texte. L'accompagnement des élèves dans la tâche devrait ainsi leur permettre de participer à l'avancement de l'énigme didactique en produisant des réponses justes ; notre hypothèse est que nous devrions observer un degré de réussite ni très faible ni très important, qui serait le gage d'une implication du plus grand nombre dans la réalisation de la tâche... même si ces réussites durant la séance sont le résultat d'une révision à la baisse des exigences de l'enseignant. Les enseignants auraient ainsi construit des schèmes relatifs au niveau de difficulté des questions qu'ils posent à leurs élèves.

\section{Méthodologie}

\subsection{Quels choix méthodologiques pour approcher des savoir-faire implicites ?}

Se pose à nous la question de l'identification des schèmes des enseignants : s'ils existent, comment les repérer ? Comme le note Vergnaud (1985), les sujets qui ont construit des schèmes n'en sont pas nécessairement conscients. Au contraire, les schèmes seraient majoritairement non explicites et difficilement verbalisables. Vergnaud (2001) prend l'exemple notoire d'un porcher qui parvient avec son bâton à conduire calmement les porcs jusqu'à l'abattoir... parce qu'il a appris, sur le tas, à repérer les porcs susceptibles d'avoir des problèmes cardiaques, et à les guider jusqu'à l'entrée de l'abattoir avant les autres, afin que le troupeau suive leur rythme. Il ne sait toutefois expliciter ni les indices qu'il prend, ni les raisons de ses gestes. Les professionnels plus qualifiés ne sont pas davantage conscients de leurs compétences : lorsqu'il est demandé à des ingénieurs experts dans la conception de lanceurs spatiaux de restituer leurs savoir-faire dans des guides méthodologiques destinés aux jeunes ingénieurs, seule une faible partie de ces savoir-faire est évoquée. Il existe dans toutes les professions un décalage entre la forme prédicative et la forme opératoire de la connaissance : il est possible de savoir dire sans savoir faire, mais aussi de savoir faire sans savoir dire (Vergnaud, op. cit.).

Ainsi, nous suivons Rogalski (2007, p. 164) lorsqu'elle écrit que « pour parler d'un schème d'action d'un sujet, cela suppose qu'on ait observé son activité dans un certain nombre de situations d'une même classe et qu'on ait pu dégager un invariant dans l'organisation de cette activité ».

C'est donc l'observation de l'activité d'enseignants dans plusieurs situations similaires que nous privilégions pour mettre en évidence les éventuels schèmes qu'ils auraient construits.

\subsection{Mise en place du dispositif méthodologique}


Nous avons observé 35 séances de découverte de texte au Cours Préparatoire : 1 séance $^{2}$ par mois entre décembre et juin, dans 5 classes «ordinaires» dont les enseignants sont expérimentés (entre 11 et 18 ans d'expérience d'enseignement au primaire; entre 5 et 9 ans d'expérience d'enseignement au CP). Nous nous centrons sur une dimension qui n'est pas enseignée formellement aux professeurs, et qui est donc potentiellement associée à un savoirfaire construit implicitement : il s'agit de poser des questions aux élèves qui aboutissent à un «Taux de Réponses Justes » acceptable par la classe. Nous construisons l'indicateur du «Taux de Réponses Justes » (TRJ), mesure globale qui, pour une séance, s'applique à l'ensemble du groupe-classe. Il reflète le niveau de difficulté de la tâche observable lors d'une séance, en se fondant sur les performances des élèves. Pour obtenir le TRJ, nous relevons, durant une séance de classe, la succession de sous-tâches qui composent la tâche globale de lecture de texte. Elles correspondent à des questions ou des injonctions «publiques »des enseignantes. Pour déterminer si la réponse à ces sous-tâches est juste ou non, nous nous fondons sur le niveau d'exigence des enseignants : nous considérons que la réponse est juste si l'enseignant la valide (explicitement, ou implicitement en passant à la sous-tâche suivante).

Nous obtenons ainsi un tableau du type :

\begin{tabular}{|l|l|l|l|}
\hline Sous-tâche & Élève qui répond & Réponse & Juste/Faux \\
\hline $\begin{array}{l}\text { Rappelez-moi le début } \\
\text { de l'histoire. }\end{array}$ & Johan & $\begin{array}{l}\text { Martin il avait fait } \\
\text { un cauchemar. }\end{array}$ & Juste \\
\hline $\begin{array}{l}\text { Le titre. Léa, qu'est-ce qu'il } \\
\text { y écrit? }\end{array}$ & Léa & Martin & Faux \\
\hline $\begin{array}{l}\text { De quoi ça va parler, } \\
\text { ce texte? }\end{array}$ & Suleymane & $\begin{array}{l}\text { De Martin quand il prend } \\
\text { son petit-déjeuner. }\end{array}$ & Juste \\
\hline$\ldots$ & $\ldots$ & $\ldots$ & $\ldots$ \\
\hline
\end{tabular}

Exemple de succession de sous-tâches et des réponses associées

Le Taux de Réponses Justes est ainsi le pourcentage de sous-tâches réalisées sans indication supplémentaire et validées par l'enseignant. Cet indicateur inclut les réponses justes obtenues par effets Topaze et Jourdain (Brousseau, 1998), où l'enseignant effectue la plus grande partie du travail à la place de l'élève, mais où l'élève produit une réponse validée par l'enseignant.

\section{Résultats : les enseignants expérimentés obtiennent un Taux de Réponses Justes viable pour la classe}

Pour chacune des trente-cinq séances observées, nous avons analysé les interactions didactiques verbales enseignant-élèves et relevons le Taux de Réponses Justes. Les résultats obtenus sont illustrés par le graphique ${ }^{3}$ suivant. Dans ce graphique, chaque mois, les 5 points correspondent aux Taux de Réponses Justes (TRJ) des séances observées dans chacune des 5 classes (A, B, C, D et E).

\footnotetext{
${ }^{2}$ Les séances observées durent entre 23 minutes et 1 heure 3 minutes.

${ }^{3}$ Les lignes reliant les points n'ont pas de signification; elles permettent seulement de visualiser plus aisément les points relatifs à une même classe.
} 


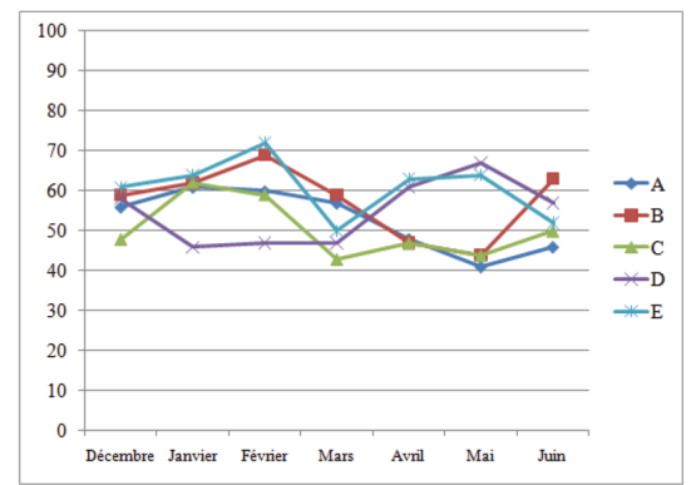

Taux de Réponses Justes, en fonction de la classe (A, B, C, D, E) et du mois

Nous constatons que les Taux de Réponses Justes sont peu variés, quels que soient la classe, la période de l'année, le mode de groupement des élèves, l'accompagnement de l'enseignant, etc. Ils se situent dans un cadre n'excédant pas $16 \%$ autour de la médiane, égale à $57 \%$ : dans aucune des séances observées le TRJ n'est inférieur à $41 \%$ ou supérieur à $72 \%$ (valeurs extrêmes).

Par les questions qu'ils posent (découpage de la tâche globale de lecture en sous-tâches) et les élèves qu'ils choisissent d'interroger, les enseignants parviennent donc à maintenir un TRJ quasi constant au fur et à mesure de l'année scolaire : bien que les élèves soient de plus en plus performants en lecture, les questions qui leur sont posées ne provoquent pas de plus en plus de réussite. Jamais la classe ne se trouve en situation d'échecs répétés, ou de nombreuses réussites successives. Ce résultat confirme que les enseignants savent anticiper et ajuster le niveau de difficulté des tâches (Maurice, 1996).

Nos données montrent que cette stabilité est obtenue grâce à deux types de régulations :

- une différenciation inter-séances, c'est-à-dire, à différents moments de l'année scolaire, une adaptation de la complexité des sous-tâches au niveau de la classe. La décomposition de la tâche initiale (lire un texte) en sous-tâches (les questions auxquelles les élèves ont à répondre) n'est pas de même type au fur et à mesure de l'année : une analyse qualitative montre que la complexité des sous-tâches augmente avec le niveau des élèves en lecture. Par exemple, dans une des classes observées, plus l'année avance, plus l'enseignante demande aux élèves de répondre à des questions de compréhension alors que le texte n'a pas encore été oralisé publiquement (seuls les élèves qui ont su lire le texte peuvent y répondre, alors qu'en début d'année, même les non-lecteurs pouvaient répondre aux questions de compréhension qui portaient sur ce qui avait déjà été oralisé par un autre élève).

- une différenciation intra-séance, c'est-à-dire une adaptation du niveau de difficulté des sous-tâches au niveau de chaque élève interrogé. Lors d'une même séance, si les sous-tâches étaient adressées indifféremment aux élèves de divers niveaux, les plus faibles auraient peu d'opportunités de réussir (répondre juste), tandis que les élèves forts répondraient quasi systématiquement correctement. Afin que la séance soit viable pour l'ensemble de la classe, les enseignants n'interrogent pas les élèves au hasard pour répondre à une sous-tâche donnée. Nos analyses mettent en évidence que les sous-tâches les plus complexes s'adressent aux élèves les plus performants (par exemple, ces derniers sont plus souvent interrogés pour lire des phrases entières que des mots isolés). Ainsi, le Taux de Réponses Justes des élèves les plus faibles n'est pas significativement moins élevé que celui des autres élèves.

Obtenir un Taux de Réponses Justes acceptable par la classe et par chacun des élèves n'est pas enseigné aux professeurs et découle donc d'un apprentissage informel, se construisant dans l'action. 


\section{Conclusion}

Nous avons interrogé dans cette contribution les choix des enseignants lorsqu'ils proposent à leurs élèves une tâche d'apprentissage sans évaluation formelle (ici, la lecture d'un texte en Cours Préparatoire). Nous avons montré que des observations répétées de séances d'enseignement pouvaient conduire à mettre au jour des «organisations invariantes de l'activité » (Vergnaud, 2001), signes de schèmes construits par les enseignants pour piloter leur classe. Nos résultats montrent que les questions posées aux élèves, qui marquent l'avancée dans la résolution de cette tâche, permettent à l'enseignant de piloter sa classe, le Taux de Réponses Justes obtenu paraissant acceptable à la fois de la part de la classe et de chacun des élèves. Pour parvenir à un Taux de Réponses Justes ni très faible ni très élevé, de la part de chaque élève de la classe, les interactions enseignant-élèves ne sont pas de même nature selon le niveau des élèves : les enseignants tendent à adapter leur accompagnement au niveau de leurs élèves. Une analyse qualitative des questions auxquelles sont confrontées les élèves faibles montre que les gestes d'ajustement (Jorro, 2007), bien que nécessaires, se révèlent correspondre à un «sur-ajustement aux difficultés et aux caractéristiques des élèves » (Bautier \& Rochex, 2004, p.14); avec une approche théorique et méthodologique différente, nous parvenons aux mêmes conclusions que ces derniers auteurs, qui écrivent que les enseignants sont conduits, "par souci de faciliter leur réussite et de préserver leur image de soi, à proposer [aux élèves] des tâches simplifiées à l'excès, morcelées et ne faisant appel qu'à des compétences cognitives "de bas niveau" et à des situations "fermées", dans lesquelles l'activité des élèves est pour l'essentiel déterminée et contrôlée de l'extérieur, par les procédures et les consignes et/ou par l'enseignant »(Bautier \& Rochex, op.cit.).

Goigoux (1998) effectue des observations comparables lors de séances de lecture en SEGPA ${ }^{4}$ : les enseignants permettent aux élèves « de préserver une image acceptable d'eux-mêmes en valorisant leurs productions et en procédant à de très nombreux feed-back positifs. Ils les encouragent et évitent de les accabler, quitte à simplifier les tâches pour permettre à chacun d'obtenir des résultats honorables ». C'est un constat similaire qu'effectue Bonnéry (2009), en analysant des séances de mathématiques en Cours Moyen 2e année : «les élèves sont (...) "disposés", de manière plus ou moins implicite, à la fois dans une hiérarchie scolaire et dans un dispositif et un cadre de travail apparemment collectifs, qui permet aux plus faibles de réussir des tâches, voire d'être valorisés pour cela, sans pour autant opérer le cheminement intellectuel attendu, de participer au travail collectif sans pour autant réellement apprendre ».

Nos résultats ont l'avantage de montrer quantitativement, à partir d'un grand nombre de séances $(\mathrm{N}=35)$ et plusieurs enseignants $(\mathrm{N}=5)$, que ces constats qualitatifs sont récurrents.

Un Taux de Réponses Justes constant, ni très faible ni très élevé, répondrait à la nécessité de maintenir les élèves dans la relation didactique (Amade-Escot, 2007) ; les enseignants ont appris, de façon informelle, à interroger les élèves en fonction de la réponse attendue. L'ajustement des sous-tâches (questions posées) au niveau des élèves et à la période de l'année conduit à considérer les sous-tâches comme instruments de pilotage de la classe (Rabardel, 1995 ; Maurice, 1996). Elles auraient notamment pour fonction de maintenir un climat viable, propice à la participation de chacun dans le jeu d'enseignement-apprentissage. Cette organisation invariante de l'activité corrobore l'hypothèse selon laquelle les enseignants construisent des schèmes d'utilisation des tâches proposées aux élèves. Ces schèmes, en grande partie implicites, se construisent de manière informelle pendant l'action.

\footnotetext{
${ }^{4}$ Section d'Enseignement Général et Professionnel Adapté.
} 
Il va de soi que l'ensemble des savoir-faire des enseignants expérimentés ne peut être explicité. Leur explicitation comporte toutefois plusieurs enjeux. Tout d'abord, elle permet de mieux expliquer et comprendre les pratiques enseignantes, les contraintes qui les mettent en forme, et par là même les contraintes qui cadrent les opportunités d'activité des élèves. Ces savoir-faire ont tout intérêt à être conscientisés par les enseignants qui les mettent en œuvre ; en effet, cette mise au jour autorise à considérer ces « organisations invariantes de l'activité » comme des leviers d'action pour tenter de produire des effets souhaités en termes d'apprentissages et de gestion de la classe.

Dans cette recherche (Murillo, 2009, 2010), les savoir-faire des enseignants ont été mis au jour à partir d'un point de vue extrinsèque, celui du chercheur, qui a pris pour intermédiaire l'observation des interactions entre enseignant et élèves pour en inférer les schèmes des enseignants. Dans une recherche en cours, nous adjoignons un point de vue intrinsèque, celui de l'enseignante observée, qui explicite certaines de ses préoccupations lors d'un entretien, par exemple : «ce à quoi je pensais beaucoup, c'était de faire participer ces enfants-là qui sont à la traîne, et leur montrer qu'ils savaient des choses ». L'analyse de ces entretiens nous permettra de mieux comprendre les fonctions des questions que les enseignants posent à leurs élèves.

\section{Bibliographie}

Amade-Escot C. (2007), « Les savoirs au cœur du didactique », in Le didactique, C. AmadeEscot (dir.), Paris, Éditions Revue EPS.

Bautier E., \& Rochex J.Y. (2004), «Activité conjointe ne signifie pas significations partagées », in Situation éducative et significations, C. Moro \& R. Rickenmann (dir.), Bruxelles, De Boeck, p. 199-220.

Bonnéry S. (2009), «Scénarisation des dispositifs pédagogiques et inégalités d'apprentissage », Revue française de pédagogie, n¹67, p. 13-23.

Bressoux P., \& Pansu P. (2003), Quand les enseignants jugent leurs élèves, Paris, PUF.

Brousseau, G. (1998), Théorie des situations didactiques, textes rassemblés par Balacheff N. et al., Grenoble, La Pensée Sauvage.

Bru M. (1991), Les variations didactiques dans l'organisation des conditions d'apprentissage, Toulouse, EUS.

Carré P. (2005), L'apprenance : vers un nouveau rapport au savoir, Paris, Dunod.

Crahay M. (1996), Peut-on lutter contre l'échec scolaire?, Bruxelles, De Boeck Université.

Doyle W. (1986), « Classroom organization and management », in Handbook of Research on Teaching, M. C. Wittrock (dir.), New York, McMillan, p. 392-431.

Ducroquet R. J. (1965), La marche et les boiteries. Étude des marches normales et pathologiques, Paris, Masson et cie.

Goigoux R. (1998), «Apprentissage et enseignement de la lecture en SEGPA. Synthèse de l'étude "Les élèves en grande difficulté de lecture et les enseignements adaptés" », Ministère de l'Éducation nationale, de la Recherche et de la Technologie.

Grisay A. (1984), «Les mirages de l'évaluation scolaire. Rendement en français, notes et échecs à l'école primaire », Revue de la Direction Générale de l'Organisation des Études, $\mathrm{n}^{\circ} 5$, p. 29-42.

Jorro A. (2007), «L'évaluation, génératrice de développement professionnel ?», in Évaluation et développement professionnel, A. Jorro (dir.), Paris, L'Harmattan, p. 11-31.

Leplat J. (2000), L'analyse psychologique de l'activité en ergonomie : aperçu sur son évolution, ses modèles et ses méthodes, Toulouse, Octarès.

Leplat J., \& Hoc J. M. (1983), «Tâche et activité dans l'analyse psychologique des situations », Cahiers de Psychologie Cognitive, vol. 3, n 1, p. 49-63. 
Maurice J. J. (1996), Modélisation du savoir-faire de l'enseignant expérimenté : adaptation aux contraintes, anticipation, négociation, pilotage de la classe par les tâches scolaires, Thèse en sciences de l'éducation, non publiée, Université Pierre Mendès-France, Grenoble.

Maurice J. J. (2006), «L'expérience de l'enseignant : une réflexivité limitée », Formation et pratiques d'enseignement en questions, $\mathrm{n}^{\circ} 3$, p. 53-67.

Maurice J. J. (2007), «Modélisations de conceptualisations en acte des enseignants : une genèse instrumentale », in Activité humaine et conceptualisation. Questions à Gérard Vergnaud, M. Merri (dir.), Toulouse, PUM, p. 783-792.

Maurice J. J., \& Murillo A. (2008), «La Distance à la Performance Attendue : un indicateur des choix de l'enseignant en fonction du potentiel de chaque élève », Revue Française de Pédagogie, $\mathrm{n}^{\circ} 162$, p. 67-80.

Murillo A. (2009), Quels choix des enseignants quant au niveau de difficulté des tâches prescrites à leur classe? En lecture, au Cours Préparatoire, thèse en sciences de l'éducation, non publiée, Université de Toulouse, Toulouse.

Murillo A. (2010), «Le niveau de difficulté des tâches scolaires : des marges de manœuvre limitées pour les enseignants », Carrefours de l'Éducation, 29, p. 79-93.

Pastré P. (2011), La didactique professionnelle. Approche anthropologique du développement des adultes, Paris, PUF.

Piaget J. (1974), Réussir et comprendre, Paris, PUF.

Plas F., Viel E., \& Blanc Y. (1983), La marche humaine, Paris, Masson.

Posthumus K. (1947), Levensgehell en school, La Haye.

Rabardel P. (1995), Les hommes et les technologies. Approche cognitive des instruments contemporains, Paris, Armand Colin.

Rabardel P., Carlin N., Chesnais M., Lang N., \& Pascal M. (1998), Ergonomie: concepts et méthodes, Toulouse, Octarès.

Remoussenard P. (2014), En quête du travail caché: enjeux scientifiques, sociaux, pédagogiques, Toulouse, Octares.

Rogalski J. (2003), "Y a-t-il un pilote dans la classe ? Une analyse de l'activité de l'enseignant comme gestion d'un environnement dynamique ouvert», Recherches en Didactique des Mathématiques, 23(3), p. 343-388.

Rogalski J. (2007), «Situations et schèmes. Action et connaissance », in Activité humaine et conceptualisation. Questions à Gérard Vergnaud, M. Merri (dir.), Toulouse, PUM, p. 161178.

Savoyant A. (2008), «Quelques réflexions sur les savoirs implicites », Travail et apprentissages, 1, p. 92-100.

Sensevy G. (2007), « Vergnaud, un pragmatiste ? , in Activité humaine et conceptualisation. Questions à Gérard Vergnaud, M. Merri (dir.), Toulouse, PUM, p. 41-48.

Six F. (1999), De la prescription à la préparation du travail : apports de l'ergonomie à la prévention et à l'organisation du travail sur les chantiers de bâtiment, note de synthèse pour l'Habilitation à Diriger des Recherches, Université Charles de Gaulle Lille 3, Lille.

Tiercelin C. (2002), Hilary Putnam : l'héritage pragmatiste, Paris, PUF.

Vergnaud G. (1985), "Concepts et schèmes dans une théorie opératoire de la représentation », Psychologie française, p. 254-251.

Vergnaud G. (1996), «Au fond de l'action, la conceptualisation », in Savoirs théoriques et savoirs d'action, J. M. Barbier (dir.), Paris, PUF, p. 275-292.

Vergnaud G. (1999), «On n'a jamais fini de relire Vygotski et Piaget », in Avec Vygotski, Y. Clot (dir.), Paris, La Dispute, p. 45-58.

Vergnaud G. (2001), «Forme opératoire et forme prédicative de la connaissance », in Actes du Colloque GDM-2001. La notion de compétence en enseignement des mathématiques, 
analyse didactique des effets de son introduction sur les pratiques et sur la formation, J. Portugais (dir.), Montréal, p. 6-27.

Vergnaud G., \& Récopé M. (2000), «De Revault d'Allonnes à une théorie du schème aujourd'hui », Psychologie française, vol. 45, n ${ }^{\circ}$, p. 35-50. 